\section{Addition of Alpha-Amylase and Thickener to Blenderized Rice Provides Suitable Viscosity for Use in Nutritional Support}

Keywords: Blenderized diet; Viscosity; Temperature; Enteral feeding Abstract
Background: Blenderized diets have been widely adopted as
nutritional support, particularly for patients receiving formulae via
an enteral route. Although the viscosity of thickened formulae is a
determinant of adverse events, no study has examined the viscosity of blenderized diets.

Aim: To examine the viscosity and temperature of four types of blenderized diet.

Methods: Four types of rice porridge were prepared: rice porridge (RP) (Method 1); rice porridge with $0.5 \%$ alpha-amylase (RPA) (Method 2); rice porridge with $0.5 \%$ alpha-amylase and $0.5 \%$ thickener (RPAT) (Method 3); and rice porridge with $0.5 \%$ alpha-amylase and $0.5 \%$ thickener frozen overnight and defrosted (RPATFD) (Method 4). The viscosity and temperature of these four types of blenderized diet were measured for 30 minutes with a B-type viscometer.

Results: The viscosity of blenderized diets over 30 minutes increased with decreasing temperature with Methods 1, 3, and 4. With Method 2 , viscosity at 5 minutes was significantly lower than that with Method $1(p<0.05)$. Moreover, unlike the other three methods, viscosity with Method 2 decreased significantly over the 30 minutes $(p<0.05)$. This phenomenon might have been related to the activation of added amylase. Viscosity during the 30 minutes was within the range of 3,500-10,000 mPa.s, which is reported acceptable in clinical settings. Overnight freezing and defrost of the blenderized diet in Method 4 appeared to stably maintain the viscosity within the likely acceptable range achieved with Method 3.

Conclusion: The addition of $0.5 \%$ thickener with $0.5 \%$ alphaamylase, either alone or followed by overnight freezing followed by defrosting, appeared to stably maintain the viscosity of a blenderized diet within the range likely acceptable in clinical settings. These findings might aid in the development of clinically usable blenderized diets.

\section{Introduction}

Registered dietitians in pediatric nutritional practice often encounter healthcare issues with severely handicapped children returning home after an extended period of hospitalization. Severely disabled children have difficulty in their intellectual ability and movement. Moreover, it is essential to provide enteral feeding immediately after birth because of dysphagia accompanying impairment of the central nervous system. Therefore, the first prescription diet must often be based on enteral nutrient formulae, which might lower their QOL. In addition, the use of a liquid nutritional supplement often results in multiple clinical complications which can might impair the growth and development of the child, such as vomiting, nausea [1].

Against this background, thickened formula (TF) has been
Nutrition and Health

\author{
Mao Itoh', Yukiko Nishimoto², Hiroshi Masui', \\ Yuri Etani ${ }^{3}$, Kazuko Takagishi ${ }^{1}$, Shinobu Ida ${ }^{3}$ and \\ Teruyoshi Amagai ${ }^{*}$ \\ ${ }^{\prime}$ Master's Program, Administration Food Sciences and Nutrition \\ Major, Graduate School of Human Environmental Sciences, \\ Mukogawa Women's University, Osaka, Japan \\ ${ }^{2}$ Department of Nutrition, Osaka Medical Center and Research \\ Institute for Maternal and Child Health, Hyōgo, Japan \\ ${ }^{3}$ Department of Pediatric Gastroenterology, Nutrition and \\ Endocrinology, Osaka Medical Center and Research Institute for \\ Maternal and Child Health, Hyōgo, Japan \\ ${ }^{4}$ Department of Human Environmental Sciences, School of Human \\ Environmental Sciences, Mukogawa Women's University, Hyōgo, \\ Japan \\ *Address for Correspondence \\ Teruyoshi Amagai, Department of Human Environmental Sciences, School of \\ Human Environmental Sciences, Mukogawa Women's University, Nishinomiya, \\ Hyōgo, Japan, Tel: 81798459855; E-mail: amagait@nutrped.com \\ Submission: 12 April, 2016 \\ Accepted: 30 April, 2016 \\ Published: 05 May, 2016 \\ Copyright: ๑ 2016 Itoh M. This is an open access article distributed \\ under the Creative Commons Attribution License, which permits \\ unrestricted use, distribution, and reproduction in any medium, provided \\ the original work is properly cited.
}

widely adopted as a novel type of nutritional support, particularly in preventing adverse events in clinical settings [2-4]. Two types of TF are available in ordinary clinical settings, mainly in developed countries such as the United States [2-7], a liquid type and a blenderized diet (BD). TF with a viscosity close to that of chewed foodstuffs is reported to be absorbed and digested normally $[8,9]$. Although the viscosity of these diets is considered a determinant of adverse events in clinical settings, such as diarrhea and symptoms related to gastro-esophageal reflux disease [2-4], the reports on the viscosity of blenderized diets (BDs) has not been conducted. Teresa et al. evaluated the clinical necessity and feasibility of BDs in clinical settings via a questionnaire survey of registered dietitians (RDs) belonging to the Pediatric Nutrition Practice Group, with particular regard to clinical experience with blenderized whole foods, defined as BDs with whole diets as included in the present study [10]. More than half $(57.6 \%)$ of responding RDs considered that blenderized whole diets were the most suitable type of diet for administration via an enteral tube, and most parents $(70.2 \%)$ wished to use them, with only $6.1 \%$ of parents preferring the use of natural, non-chemical foods. This survey suggests that blenderized whole diets might be considered the leading option for patients with an enteral tube.

In clinical settings, caregivers often utilize BDs administered through an enteral tube to provide required nutrients to patients on a daily basis.

For the patients with head and neck cancers, evidences with thickened liquids are extremely limited [11]. Additionally, not thicken liquid but puree like Nectar was proved to be able to swallow 
Citation: Itoh M, Nishimoto Y, Masui H, Etani Y, Takagishi K, et al. Addition of Alpha-Amylase and Thickener to Blenderized Rice Provides Suitable Viscosity for Use in Nutritional Support. J Nutri Health. 2016;2(1): 7.
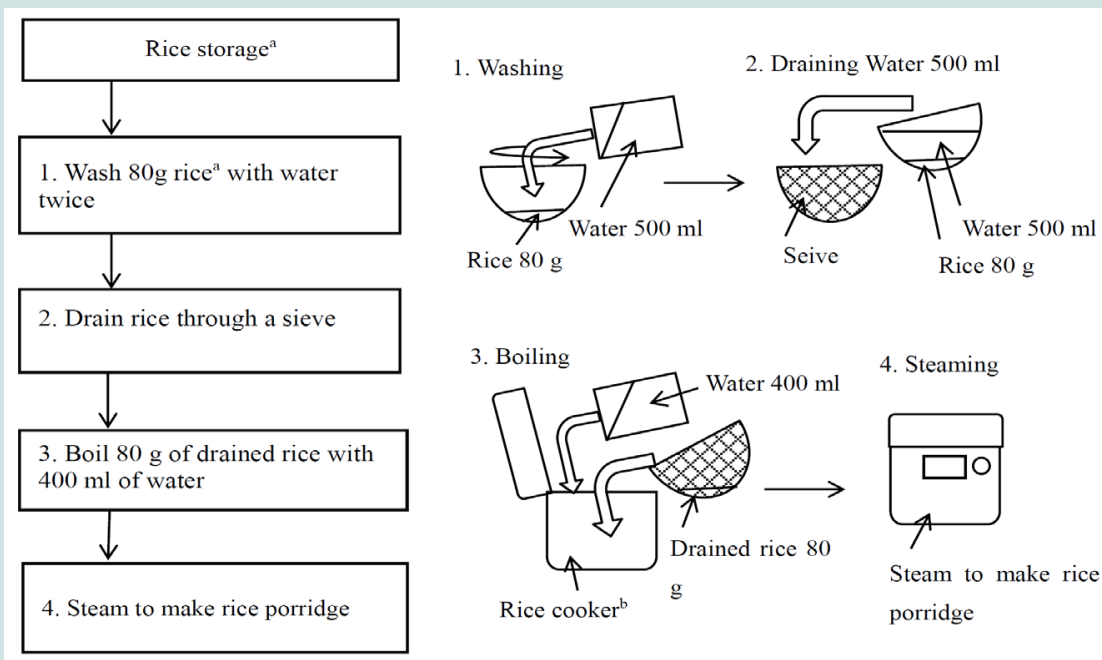

4. Steaming

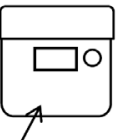

Steam to make rice porridge

Figure 1: Method of cooking rice to prepare porridge.

a) "Tamba Sasayama" Koshihikari brand rice (Hyogo Prefecture, Japan) was stored at $15{ }^{\circ} \mathrm{C}$ or lower in order maintain stable viscosity. b) Rice cooker was an RC-10 MM (Toshiba Co. Limited, Tokyo, Japan).

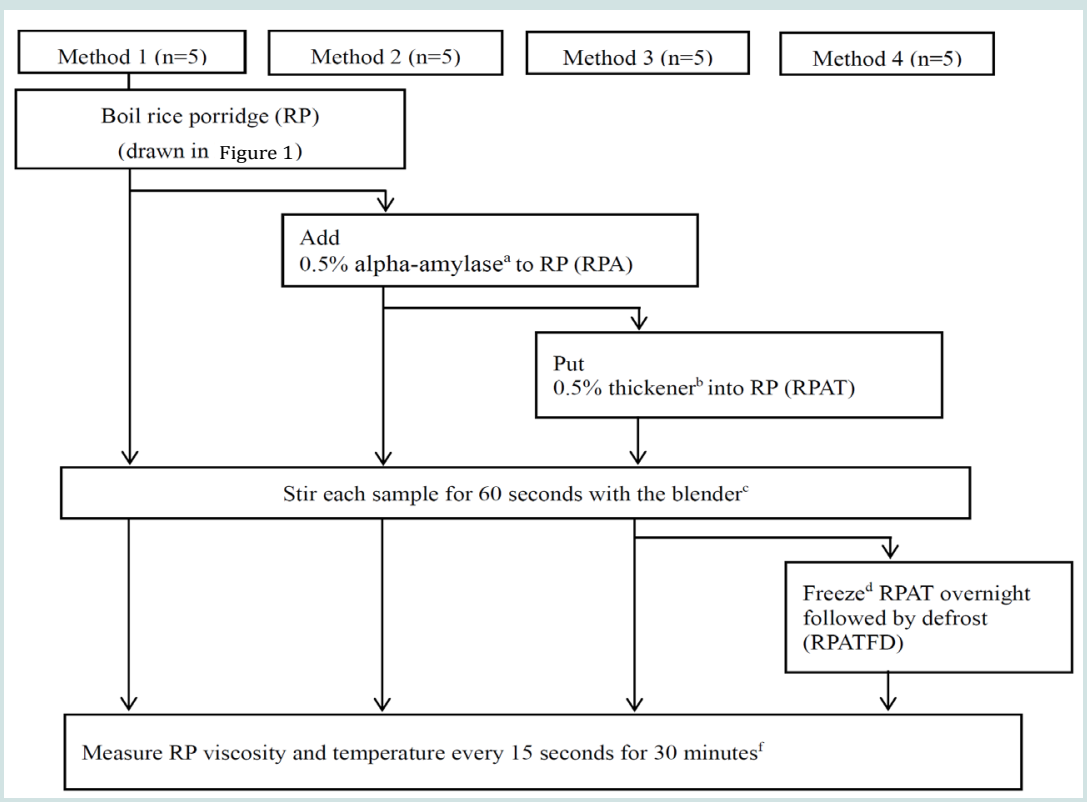

Figure 2: Study flow-chart

a) a-Amylase composition was $\alpha$ - amylase $0.2 \%$ and dextrin $99.8 \%$ (“Porridge-Helper”; Kissei Pharmaceutical Co Ltd, Nagano, Japan). b) Thickener was "Matomeruko-easy" brand composed of dextrin, glucomannan, xanthan gum, carrageenan, gellan gum, and locust bean gum (Cliniko Co. Ltd, Tokyo, Japan). c) Blender was a Braun MR730CC Multi Quick 7 cordless hand blender (De' Longhi Japan Co. Ltd, Tokyo, Japan). d) Refrigerator was R-23RA (Hitachi, Co. Ltd. Tokyo, Japan). e) Microwave oven was an NE-NS60 (Panasonic Co. Tokyo, Japan). F) Viscometer was a "Brookfield viscometer BASEPlus No.6701 (Atago Co. Ltd, Tokyo, Japan).

without aspiration [12]. However, the studies on the effect of viscosity of the diets putting directly into the stomach without swallowing in patients with swallowing difficulties are not conducted by the present study. To our knowledge, the present study is the first to examine the viscosity of BDs.

Here, to better understand the use of BDs in clinical settings, we analyzed the viscosity of various fundamental BD formulae.

\section{Methods \\ Preparation of blenderized diets}

Eighty grams of milled white rice (Tamba Sasayama Koshihikari brand; Hyogo Prefecture, Japan) was washed with $400 \mathrm{ml}$ of water at a temperature between $28-30{ }^{\circ} \mathrm{C}$, drained with a sieve, combined 
Citation: Itoh M, Nishimoto Y, Masui H, Etani Y, Takagishi K, et al. Addition of Alpha-Amylase and Thickener to Blenderized Rice Provides Suitable Viscosity for Use in Nutritional Support. J Nutri Health. 2016;2(1): 7.

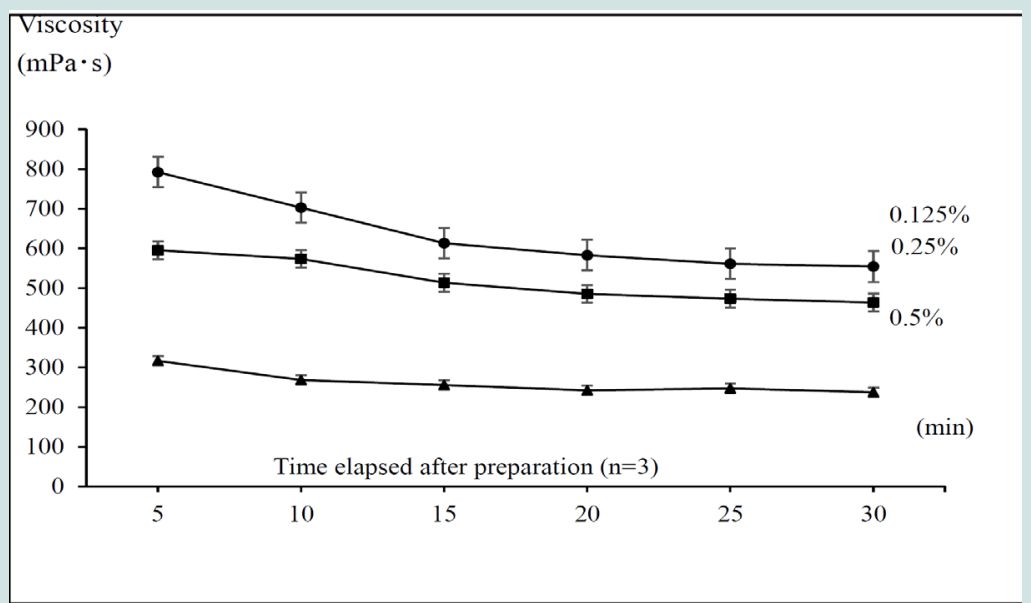

Figure 3: Preliminary experimental results (comparison of viscosity due to the addition of $0.5 \%$ alpha-amylase, $0.25 \%$ alpha-amylase, and $0.125 \%$ alpha-amylase.) All data are presented as the mean \pm standard deviation (SD).

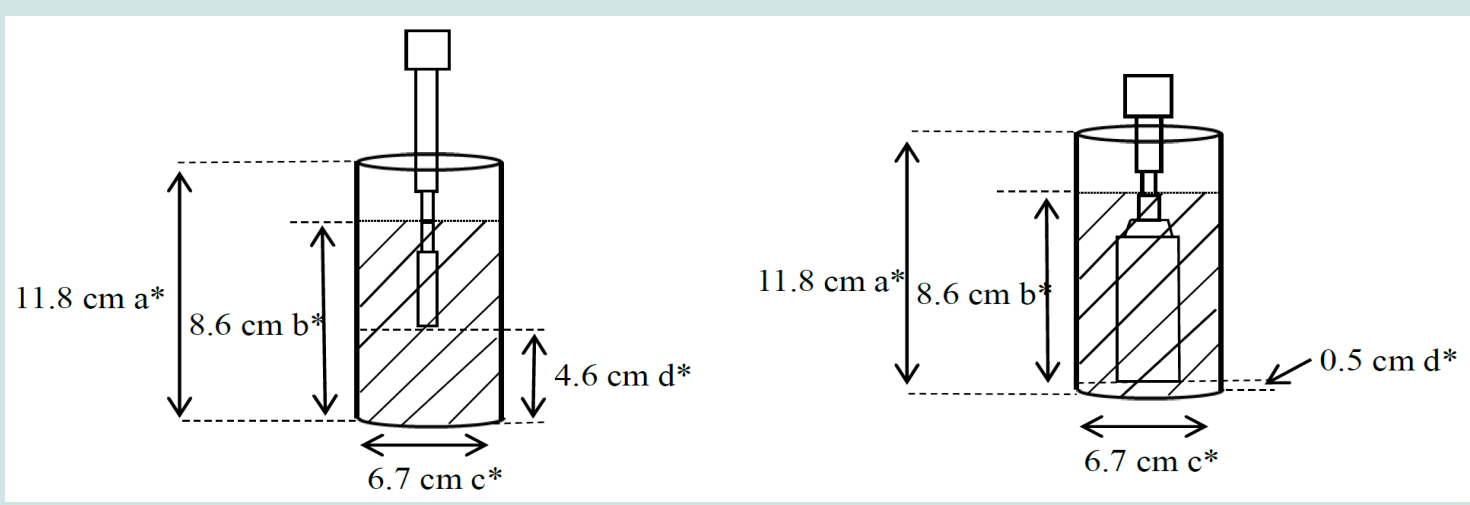

Figure 4: Experimental condition of the rotor and container

a) Figure on the left shows the L1 rotor used in Methods 1, 3, and 4. b) Figure on the right shows the L4 rotor used in Method 2.

$a^{*}$ : Height of the container, $b^{*}$ : Height to the liquid surface of the sample, $c^{*}$ : Diameter of the container, $d^{*}$ : Distances between the bottom of the rotor and the bottom of container.

with a further $400 \mathrm{ml}$ water and cooked by boiling with a rice cooker ("RC-10MM", Toshiba Corporation, Tokyo, Japan). Cooking was conducted using the automatic setting of the rice cooker, under which the rice was soaked for 30 minutes to absorb water followed by steaming for 75 minutes (Figure 1). The rice was stored at $15{ }^{\circ} \mathrm{C}$ before use according to a previous report [13].

\section{Four types of rice porridge to examine}

The four methods below were designed to determine whether the various types of rice porridge could be prepared within the viscosity and temperature ranges acceptable for easy and safe delivery to patients through an enteral tube. Following preparation using Methods 1-4, the viscosity and temperature of the four types of rice porridge were measured by stirring with a blender (Braun MR730CC Multi Quick 7 cordless hand blender, De' Longhi Japan Corporation, Tokyo, Japan).

\section{Method 1}

Rice porridge (RP): Boiled rice porridge (RP, Figure 1) was stirred with the blender using a stirring speed of 5,400 $\pm 1,000$ rate per minute (rpm). The viscosity and temperature of the RP were then measured every 15 seconds for the next 30 minutes.

\section{Method 2}

Addition of $\mathbf{0 . 5 \%}$ alpha-amylase to RP (RPA): To ensure $\mathrm{RP}$ reached the reported target viscosity range (7), $0.5 \mathrm{w} / \mathrm{w} \%$ of alpha-amylase was added to the RP and the mixture was stirred for 60 seconds with the blender (Figure 2). In this study, the $0.5 \%$ concentration was adopted based on the experimental preliminary finding on the addition of $0.125 \%, 0.25 \%$, or $0.5 \%$ concentrations of alpha-amylase to RP that $0.5 \%$ had a standard deviation less than $0.5 \%$ (Figure 3). Alpha-amylase (Kissei Pharmaceutical Company Limited, Nagano, Japan) is composed of alpha-amylase $0.2 \%$ and dextrin $99.8 \%$. Alpha-amylase products were selected based on their easy market availability as an enzymatic product for food adjustment in nursing care settings. 


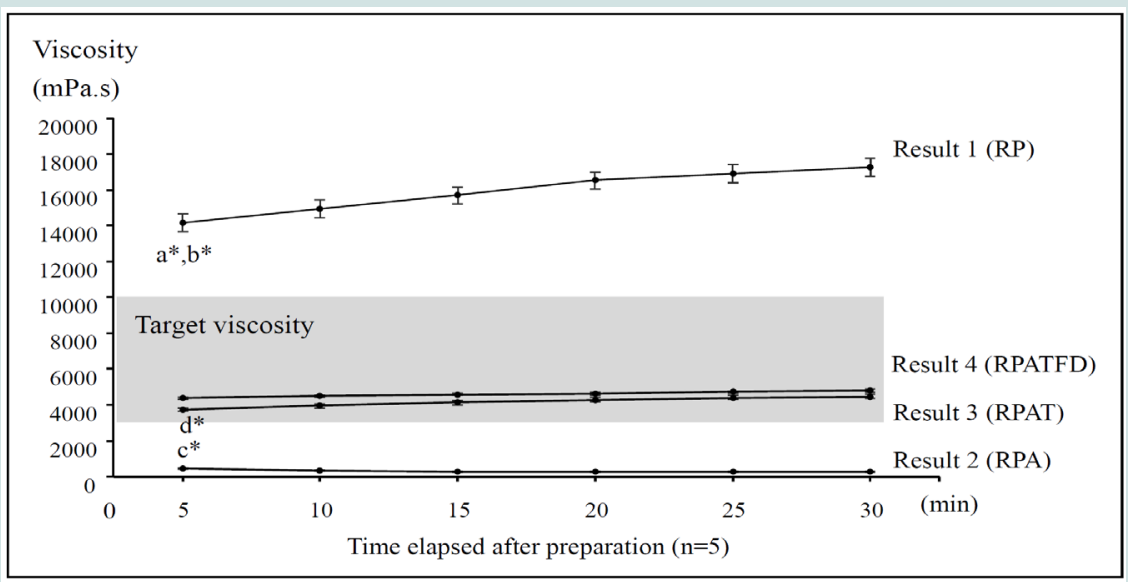

Figure 5: Viscosity changes for 30 minutes after blenderized diet preparation

All data are presented as the mean \pm standard deviation (SD). Time zero (0) was defined as the time immediately after the preparation of materials in Methods 1 to 4. The shaded area shows target range of viscosity of materials, between 3,500 and $10,000 \mathrm{mPa}$.s, reported clinically acceptable (9). All data are presented as the mean \pm standard deviation (SD). Time zero (0) was defined as the time immediately after the preparation of materials in Methods 1 to 4 . The shaded area shows target range of viscosity of materials, between 3,500 and $10,000 \mathrm{mPa}$.s, reported clinically acceptable ( 9 ). a*: P <0.01 (5 minutes vs. 25 minutes, $14145 \pm 3046$ vs. $16905 \pm 3369$, respectively), b*: $\mathrm{P}<0.05$ ( 5 minutes vs. 30 minutes, $14145 \pm 3046$ vs. $17274 \pm 3481$, respectively), $c^{*}$ : $P<0.05$ (5 minutes vs. 30 minutes, $435 \pm 161$ vs. $259 \pm 26$, respectively), $d^{*}: P<0.05$ ( 5 minutes vs. 30 minutes, $3739 \pm 329$ vs. $4464 \pm 322$, respectively).

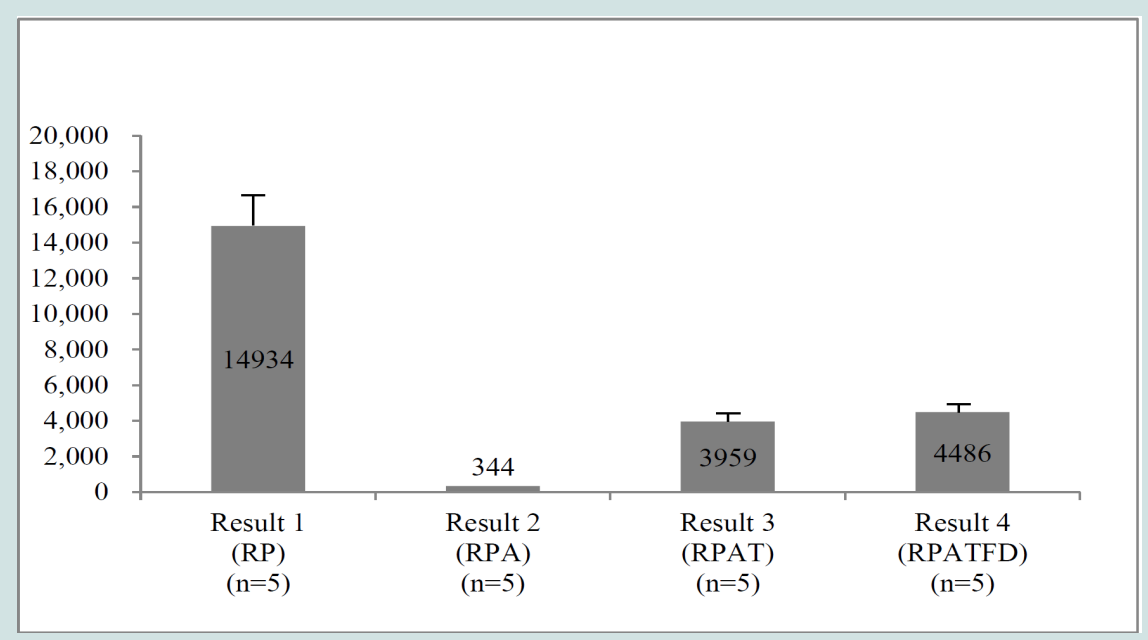

Figure 6: Comparison of viscosities following the addition of $0.5 \%$ alpha-amylase (RPA, result 2 ), addition of $0.5 \%$ alpha-amylase followed by $0.5 \%$ thickener (RPAT, result 3), and freezing and defrosting of RPAT (RPATFD; result 4).

Data are presented mean \pm standard deviation. Data were selected as the value at 30 minutes, because comparing the viscosity at the end of measurement. *: $P$ $<0.05$, n.s: not significant

Comparing the viscosity at the end of measurement *: P $<.05$, n.s: not significant.

\section{Method 3}

Addition of $\mathbf{0 . 5 \%}$ thickener to materials used in Method 2 (RPAT): To ensure that the viscosity of materials used in Method 2 reached the previously reported target range [14], $0.5 \mathrm{w} / \mathrm{w} \%$ thickener (Cliniko Company Limited, Tokyo, Japan) was added to RPA, and the mixture was stirred for 60 seconds as in Method 2 (Figure 2). Components of this thickener were dextrin, glucomannan, xanthan gum, carrageenan, gellan gum, and locust bean gum. This thickener was selected because of its heating-free characteristics and wide use in clinical settings, especially in home-nursing care.

\section{Method 4}

Effect of freezing and defrosting on the material used in Method 3 (RPATFD): To ensure these observations reflected ordinary clinical settings, in which blended diets are frozen for storage and then defrosted before via enteral tube delivery, we also evaluated these materials under a freeze/defrost cycle. RPAT used in Method 3 was frozen overnight followed by defrosting. Freezing conditions were as follows: storage for 6 hours at $-12{ }^{\circ} \mathrm{C}$ in a domestic freezer (R-23RA, Hitachi, Co. Ltd. Tokyo, Japan), followed by defrosting by warming in a microwave oven (NE-NS60, Panasonic Co. Tokyo, Japan) at 500 
Citation: Itoh M, Nishimoto Y, Masui H, Etani Y, Takagishi K, et al. Addition of Alpha-Amylase and Thickener to Blenderized Rice Provides Suitable Viscosity for Use in Nutritional Support. J Nutri Health. 2016;2(1): 7.

ISSN: 2469-4185

W for 4 minutes. Viscosity and temperature were then measured every 15 seconds for the next 30 minutes.

\section{Measurement of viscosity and temperature}

Viscosity and temperature were measured using a B-type viscometer (Brookfield Viscometer BasePlus No. 6701, Corporation Atago Headquarters, Tokyo, Japan) every 15 seconds for 30 minutes. For instance, average viscosity and temperature value at 5 minutes were presented as the average of 5 measurements. Viscosity and temperature at 0 minutes are not described, because recording started at 5 minutes. Measurement was conducted under the following conditions: room temperature $26-28^{\circ} \mathrm{C}$, sample amount $200 \mathrm{ml}$, glass container height $11.8 \mathrm{~cm}$ and diameter $6.7 \mathrm{~cm}$. Measuring position of the sample and rotor is shown in Figure 4. The range of rotational speed and rotor torque were checked at the start and end of each measurement to ensure that they met the acceptable range of $15 \%$ to $100 \%$ of the validated measurement range. Accordingly, we set torque speed at $20 \mathrm{rpm}$ for Methods 1, 3 and 4, and $4 \mathrm{rpm}$ for Method 2. The torque speed and rotor in Method 2 must be decreased from 20 to 4 rpm in order to ensure torque range is within $15 \%$ and $100 \%$.

\section{Statistical analysis}

Relationship between viscosity and temperature was measured by correlation analysis and expressed at 5-minute intervals for up to 30 minutes. Results are shown as the mean \pm standard deviation. Changes in viscosity and temperature over time were tested using the Friedman nonparametric test. Change in viscosity between samples was measured using the Kruskal-Wallis non-parametric test. Temperature and viscosity were correlated using Spearman's correlation coefficient for non-parametric variables. Differences of $\mathrm{p}$ $<0.05$ were considered statistically significant.

\section{Results}

\section{Results 1}

Rice porridge (RP): Viscosity of RP was significantly higher at 5 minutes than at 25 minutes or 30 minutes, $(\mathrm{P}<0.01)$ (Figure 5$)$. Temperature of RP was also significantly higher at 5 minutes than at 25 minutes or 30 minutes $(\mathrm{P}<0.05)$ (Table 1$)$. We speculate that these results represent the phenomenon of "higher viscosity with lower temperature".

\section{Results 2}

Addition of $0.5 \%$ alpha-amylase to RP (RPA): Viscosity of $\mathrm{RPA}$ at 30 minutes was significantly lower than that at 5 minutes $(\mathrm{P}<0.05)$ (Figure 5). This decreasing tendency was observed with Method 2 only. Viscosity measured at 30 minutes with Method 2 was significantly lower than that at 5 minutes $(\mathrm{P}<0.05)$ (Figure 5$)$. The temperature of RPA measured at 30 minutes also significantly lower than that at 5 minutes $(\mathrm{P}<0.01)$ (Table 1$)$. Viscosity appeared to decrease as temperature decreased. This positive relation between viscosity and temperature was observed only with Method 2 .

\section{Results 3}

Addition of $0.5 \%$ thickener to materials used in Method 2 (RPAT): Viscosities of RPAT at 30 minutes was significantly higher than that at 5 minutes $(\mathrm{P}<0.05)$ (Figure 5). The temperature of the experimental material (RPAT) decreased with time, similarly to the findings with other methods (Table 1).

\section{Results 4}

Effect of freezing and defrosting on the material used in Method 3 (RPATFD): Contrary to our expectation, the viscosity and temperature of RPAT remained stable after overnight freezing and defrosting, and did not significantly change during the 30-minute observation period. This result might show that overnight freezing of RPAT material stabilized its viscosity within the acceptable range in clinical settings for at least 30 minutes.

The results of Method 3 and Method 4 did not significantly differ, at least during the first 10 minutes (Figure 6).

\section{Correlation analysis of viscosity and temperature}

Correlation coefficients between the viscosity and temperature of materials in Methods 1, 2, 3, and 4 were $\mathrm{p}<0.001, \mathrm{R}=-0.644 ; \mathrm{p}<0.001$, $\mathrm{R}=0.794 ; \mathrm{p}=0.59 ; \mathrm{R}=-0.328$, and $\mathrm{p}<0.001, \mathrm{R}=0.624$, respectively. These negative Rs in Methods 1 and 3 show the negative correlation that viscosity increases as temperature decreases. In contrast, a positive correlation $\mathrm{R}$ means that viscosity decreases as temperature decreases, as observed in Methods 2 and 4.

\section{Discussion}

Although the use of thickened enteral formulae has been reported to lower the incidence of adverse events, such as diarrhea, vomiting and weight loss [2-4], a mechanism to explain these nutritional advantages has not been described. One possibility is that viscosity

Table 1: The temperature of materials changes for 30 minutes after blenderized diet preparation.

\begin{tabular}{|c|c|c|c|c|}
\hline $\begin{array}{l}\text { Time elapsed } \\
\text { (minutes) }\end{array}$ & $\begin{array}{c}\text { Result } 1(\mathrm{RP}) \\
\text { Temperature }\left({ }^{\circ} \mathrm{C}\right) \\
(\mathrm{n}=5)\end{array}$ & $\begin{array}{c}\text { Result } 2 \text { (RPA) } \\
\text { Temperature }\left({ }^{\circ} \mathrm{C}\right) \\
(n=5)\end{array}$ & $\begin{array}{c}\text { Result } 3 \text { (RPAT) } \\
\text { Temperature }\left({ }^{\circ} \mathrm{C}\right) \\
(\mathrm{n}=5)\end{array}$ & $\begin{array}{c}\text { Result } 4 \text { (RPATFD) } \\
\text { Temperature }\left({ }^{\circ} \mathrm{C}\right) \\
(n=5)\end{array}$ \\
\hline 5 & $57.6 \pm 2.1 \mathrm{a}^{*}, \mathrm{~b}^{*}$ & $41.4 \pm 7.1 \mathrm{c}^{*}$ & $30.1 \pm 2.5 \mathrm{~d}^{*}, \mathrm{e}^{*}$ & $30.3 \pm 6.0$ \\
\hline 10 & $55.6 \pm 2.0$ & $39.2 \pm 6.3$ & $29.9 \pm 2.4$ & $30.0 \pm 5.6$ \\
\hline 15 & $53.5 \pm 1.9$ & $37.4 \pm 5.9$ & $29.7 \pm 2.4$ & $29.7 \pm 2.4$ \\
\hline 20 & $51.3 \pm 2.4$ & $38.1 \pm 2.4$ & $29.5 \pm 2.4$ & $29.4 \pm 2.4$ \\
\hline 25 & $49.3 \pm 2.4$ & $36.8 \pm 2.4$ & $29.3 \pm 2.4$ & $29.1 \pm 2.4$ \\
\hline 30 & $47.4 \pm 2.4$ & $36.0 \pm 2.4$ & $29.2 \pm 2.4$ & $28.9 \pm 2.4$ \\
\hline
\end{tabular}

Time zero (0) was defined as the time immediately after the preparation of materials in Methods 1 to 4 . Data are presented as the mean \pm standard deviation (SD). $a^{*}: P<0.05$ ( 5 minutes vs. 25 minutes ), b*; $P<0.05$ ( 5 minutes vs. 30 minutes), $C^{*}$ : $P<0.01$ ( 5 minutes vs. 30 minutes), $d^{*}: P<0.05$ ( 5 minutes vs. 25 minutes ), $\mathrm{e}^{*}: \mathrm{P}<0.05$ ( 5 minutes vs. 30 minutes). 
Citation: Itoh M, Nishimoto Y, Masui H, Etani Y, Takagishi K, et al. Addition of Alpha-Amylase and Thickener to Blenderized Rice Provides Suitable Viscosity for Use in Nutritional Support. J Nutri Health. 2016;2(1): 7.

is raised: to prevent adverse events with non-thickened ordinary formulae. In clinical settings, caregivers often utilize blenderized whole diets delivered through an enteral tube to provide nutrients for patients [5-7]. Higher or lower viscosity BDs might be considered unsuitable, for instance due to the difficulty entailed in pushing higher viscosity diets through an enteral tube and more frequent tube blockage. In contrast, excessively low-viscosity diets might induce diarrhea or vomiting, leading to the failure to provide nutritional support. Accordingly, optimum viscosity might lie within a narrow range, such as between the 3,500-10,000 $\mathrm{mPa}$.s reported to prevent adverse events [14]. Although blenderized whole diets are mainly used in clinical settings, particularly in home care, we chose a simple diet instead of a whole diet with a naturally heterogeneous composition [5-7] to better illustrate the basic principal underlying the viscosity of blenderized whole diets. We selected rice porridge as suitable for this purpose.

The results of Method 1 can be summarized to state that the viscosity of simply prepared rice porridge is markedly higher than the $10,000 \mathrm{mPa}$.s reported as the upper limit of the acceptable range (AR) of 3,500-10,000 mPa.s in clinical settings [14]. We then added Method 2 to lower viscosity to the acceptable range. Contrary to our expectations, however, results exceeded the lower limit of AR (Figure 5). Method 3 was therefore included, in which thickener was added to the material used in Method 2. The results observed with Method 3 appear within the acceptable AR (Figure 5). For Method 4, the experimental material used in Method 3 was frozen overnight and then defrosted to reflect home care settings. Method 4 also met the AR (Figure 5). These results can be summarize in three ways: (1) the viscosity of ordinary rice porridge might be higher than the AR; (2) RP, with $0.5 \%$ alpha-amylase, appears lower than the AR; and (3) RP with alpha amylase and a $0.5 \%$ thickener with freezing and defrosting appeared to meet the AR. Method 4 might be suitable for use in clinical settings.

We also measured the temperatures of the experimental materials in all four methods, given the effect of temperature to decrease viscosity [15]. Although the mechanism of this effect of temperature on viscosity is unknown, the added amylase in Methods 2, 3, and 4 might have played a role. With Method 2, where the viscosity and temperature decreased with time elapsed contrary to the other three methods, amylase contained in the materials is thought to be activated, resulting in the decreasing tendency in viscosity (Table 1). For Methods 1,2, and 3, the temperature and viscosity of experimental materials differed significantly between at the timing of 5 minutes and 30 minutes, whereas they did not differ in Method 4 (Figure 5, Table 1 ), suggesting that the temperature of the $\mathrm{BDs}$ might a determinant of viscosity. To our knowledge, this is the first report to have proved the relation between the viscosity and temperature of the four different types of RP as the simplest models of BDs.

Another aspect of these findings should be considered. In Method 4 , the viscosity of the material did not change according to changes in temperature, whereas their viscosity did not change significantly over time of experimental period (Figure 5 and Table 1). This observation might be interpreted to mean that the freeze/defrost cycle stabilized viscosity, allowing it to meet the $\mathrm{AR}$ for clinical settings. The mechanism of this effect warrants further investigation.
Several limitations of the present study should be mentioned. First, the physiological and nutritional composition of the experimental material was simplified to facilitate analysis. In clinical practice, however, more complicated BDs are administered. The viscosity and clinical outcomes with BDs should also be analyzed. Second, although viscosity of the material in all four methods was measured after stirring, stirring conditions, such as speed and torque, differed between Method 2 and the others, because the measurement range depended on these conditions being met within the narrow range of the rheometer used, namely the viscometer type B. As different conditions might show different results, further studies should examine different viscometers and different types of $\mathrm{BDs}$, which would in turn reveal the physiological and nutritional characteristics of a wide range of BDs. Such studies should include careful recording of observations result of BDs viscosity, including viscosity measurement conditions, such as type of viscometer. Third, experimental numbers for each method were limited. Although an optimum number might be difficult to determine, and would be subject to financial, human, and time limitations, more examinations would provide stronger evidence. Fourth, the study of the change of $\mathrm{pH}$ of the materials was not conducted, whereas the effects of changes of $\mathrm{pH}$ of the materials must influence on their changes of the viscosity [16]. These studies also must be conducted in further investigations.

\section{Conclusion}

In this study, we prepared four types of blenderized diet to measure their viscosity and temperature for 30 minutes after preparation. These four types were as follows: (1) in Methods 1, 3, and 4 , the viscosity of the experimental BDs increased over the 30 minutes consistent with the decrease in temperature of the material. (2) In Method 2, the viscosity of BDs at 30 minutes was significantly lower than that observed in Method 1. (3) Also in Method 2, unlike with the other three methods, viscosity decreased significantly during the 30 minutes. This phenomenon might be related to activation of the added amylase. (4) Viscosity during the 30-minute observation remained within the range of 3,500-10,000 mPa.s, which has been reported to be acceptable in clinical settings. (5) Finally, the freezing overnight and defrosting of $\mathrm{BD}$ in Method 4 appeared to maintain viscosity stable within the acceptable range likely in method 3. To our knowledge, this is the first study to evaluate the viscosity and temperature of a blenderized diet. These results might provide basic information for further evaluation of the more complicated blenderized diets used in clinical practice.

\section{References}

1. Pahsini K, Marinschek S, Kahn Z, Dunitz-Scheer M, Scheer PJ (2015) Unintended side-effects of enteral nutrition support: the parental perspective: A qualitative analysis. J Pediatr Gastroenterol Nutr [Epub ahead of print].

2. Nishiwaki S, Araki H, Shirakami Y, Kawaguchi J, Kawade N, et al. (2009) Inhibition of gastroesophageal reflux by semi-solid nutrients in patients with percutaneous endoscopic gastrostomy. JPEN J Parenter Enteral Nutr 33: 513-519.

3. Shimoyama Y, Kusano M, Kawamura O, Zai H, Kuribayashi S, et al. (2007) High-viscosity liquid meal accelerates gastric emptying. Neurogastroenterol Motil 19: 879-886.

4. Simonian HP, Maurer AH, Knight LC, Kantor S, Kontos D, et al. (2004) Simultaneous assessment of gastric accommodation and emptying: studies with liquid and solid meals. J Nucl Med 45: 1155-1160. 
Citation: Itoh M, Nishimoto Y, Masui H, Etani Y, Takagishi K, et al. Addition of Alpha-Amylase and Thickener to Blenderized Rice Provides Suitable Viscosity for Use in Nutritional Support. J Nutri Health. 2016;2(1): 7.

ISSN: 2469-4185

5. Johnson TW, Spurlock A, Galloway P (2013) Blenderized formula by gastrostomy tube: A case presentation and review of the literature. Top Clin Nutr 28: 84-92.

6. Pentiuk S, O'Flaherty T, Santoro K, Willging P, Kaul A (2011) Pureed by gastrostomy tube diet improves gagging and retching in children with fundoplication. JPEN J Parenter Enteral Nutr 35: 375-379.

7. O'Flaherty T, Santoro K, Pentiuk S (2011) Calculating and preparing pureed by gastrostomy-tube (PBGT) diet for pediatric patients with retching and gagging postfundoplication. Child Obes Nutr 3: 361-364.

8. Rosenfeld L (1997) Gastric tubes, meals, acid, and analysis: rise and decline. Clin Chem 43: 837-842.

9. Minard G (2006) The history of surgically placed feeding tubes. Nutr Clin Pract 21: 626-633.

10. Johnson TW, Spurlock A, Pierce L (2015) Survey study assessing attitudes and experiences of pediatric registered dietitians regarding blended food by gastrostomy tube feeding. Nutr Clin Pract 30: 402-405.
11. Barbon CE, Steele CM (2015) Efficacy of thickened liquids for eliminating aspiration in head and neck cancer: a systematic review. Otolaryngol Head Neck Surg 152: 211-218.

12. Leder SB, Judson BL, Sliwinski, Madson L (2013) Promoting safe swallowing when puree is swallowed without aspiration but thin liquid is aspirated: nectar is enough. Dysphagia 28: $58-62$.

13. (2012) Ministry of agriculture, forestry and fisheries, management guidelines for the prevention of rice mold contamination. 19

14. Ichimaru S, Amagai T, Wakita M, Shiro Y (2012) Which is more effective to prevent enteral nutrition-related complications, high-or medium-viscosity thickened enteral formula in patients with percutaneous endoscopic gastrostomy?: a single-center retrospective chart review. Nutr Clin Pract 27: 545-552

15. Wakita M, Masui H, Ichimaru S, Amagai T (2012) Determinant factors of the viscosity of enteral formulas: basic analysis of thickened enteral formulas. Nutr Clin Pract 27: 82-90.

16. Ghorbani Gorji S, Ghorbani Gorji E, Mohammadifar MA (2015) Effect of pH on turbidity, size, viscosity and the shape of sodium caseinate aggregates with light scattering and rheometry. J Food Sci Technol 52: 1820-1824. 